\title{
Impacto do etanol e consumo de café na qualidade de sono de acadêmicos de medicina
}

\author{
The influence of ethanol and coffee on medical students'sleep quality
}

Gabriel Martinez ${ }^{1}$, Heitor Castanharo Escãno ${ }^{2}$, Maria Helena de Sousa ${ }^{3}$, Clóvis Antônio Lopes Pinto ${ }^{4}$

Martinez G, Escaño HC, de Sousa MH, Pinto CAL. Impacto do etanol e consumo de café na qualidade de sono de acadêmicos de medicina / The influence of ethanol and coffee on medical students'sleep quality. Rev Med (São Paulo). 2018 maio-jun.;97(3):267-72.

\begin{abstract}
RESUMO: Introdução: O consumo de etanol e café podem ser retratados como disruptores de sono principalmente em estudantes de medicina, o que pode prejudicar o bem estar e as atividades cotidianas desses graduandos. Objetivo: O objetivo deste estudo foi avaliar a qualidade de sono e sua possível correlação entre o consumo de etanol e café em estudantes da Faculdade de Medicina de Jundiaí. Modelo de estudo: Trata-se de um estudo transversal com 143 estudantes de medicina que responderam os questionários específicos referentes à qualidade de sono (PSQI - BR), consumo de etanol (AUDIT) e consumo de café. A associação entre essas variáveis foi avaliada através de testes de qui-quadrado e análise de regressão linear. Resultados: Os resultados demonstraram que $65,7 \%$ dos estudantes que responderam o questionário apresentaram índice de qualidade de sono ruim (PSQI - BR $>5$ pontos), $71,3 \%$ apresentaram consumo de baixo risco de etanol. A qualidade de sono apresentou correlação fraca $(\mathrm{R}=0.251)$, porém significativa $(\mathrm{p}=0.020)$ somente com o consumo de etanol. Conclusão: A presença desse resultado sinaliza a necessidade de projetos e cuidados que visem a promoção da qualidade de vida dos estudantes de medicina.
\end{abstract}

Descritores: Sono; Estudantes de medicina; Café; Etanol.

\begin{abstract}
Introduction: The comsuption of ethanol and coffee may be retracted as a sleep disruptor principally in medical students and this can compromise their welfare and daily activities. Objectives: The purpose of this study was to evaluate sleep quality and your possible correlation between comsuption of ethanol and caffeine in medical students of Faculdade de Medicina de Jundiaí. Methodology: This is a cross-sectional study with 143 medical students that answered specific questionnaires about sleep quality (Pittsburg Sleep Quality Index), alcohol comsuption (AUDIT) and caffeine comsuption. Chi-square tests and multivariate logistic regression models were used to identify statistically significant association between these variables. Results: The results showed that the prevalence of poor sleep quality (PSQI $>5$ points) was found to be $65,7 \% ; 71,3 \%$ had low risk comsuption of ethanol. There was a weak correlation $(\mathrm{R}=0.251)$, however statistically significant $(\mathrm{p}=0.020)$ only between sleep quality and ethanol comsuption. Conclusion: These results designate the necessity of projects and wariness to aim for promotion of medical student's quality of life.
\end{abstract}

Key words: Sleep; Students, medical; Coffee; Ethanol.

1. Faculdade de Medicina de Jundiaí (FMJ), Jundiaí-SP, BR. Aluno de Iniciação Científica e discente do curso de medicina. E-mail: gabs.marti95@gmail.com. ORCID: orcid.org/0000-0003-4096-435X.

2. Faculdade de Medicina de Jundiaí (FMJ), Jundiaí-SP, BR. Discente do curso de medicina e aluno colaborador de Iniciação Científica. E-mail: heitorescano@gmail.com. ORCID: orcid.org/0000-0002-3528-0941.

3. Faculdade de Medicina de Jundiaí (FMJ), Jundiaí-SP, BR. Coorientadora de Iniciação Científica e Professora Adjunta do Departamento de Saúde Coletiva. E-mail: estat@fmj.br. ORCID: orcid.org/0000-0002-1788-4653.

4. Faculdade de Medicina de Jundiaí (FMJ), Jundiaí-SP, BR. Orientador de Iniciação Científica e Professor Adjunto do Departamento de Morfologia e Patologia Básica. E-mail: coipinto@uol.com.br. ORCID: orcid.org/0000-0003-1711-0081.

Endereço de Correspondência: Gabriel Martinez. Rua das Goiabeiras, 515 - Bairro Jardim, Santo André, SP. CEP: 09090-060. 


\section{INTRODUÇÃO}

ato de dormir é um comportamento universal no qual se atribuiu durante anos o simples caráter passivo de restaurador das funções orgânicas, contudo hoje já se sabe que a saúde e o sono humano estão interligados e ambos se afetam positiva ou negativamente conforme quadro apresentado ${ }^{1,2}$. Os distúrbios de sono são comuns na população geral, acometendo aproximadamente um terço dos adultos e as queixas mais prevalentes são insônia e sonolência diurna excessiva ${ }^{3,4}$.

Os distúrbios de sono também são recorrentes na vida de estudantes de medicina em detrimento do extenso e exigente currículo da graduação, resultando em menor horas de sono por noite quando comparado à população adulta em geral e, consequentemente, resultar em algum efeito negativo em sua performance acadêmica ${ }^{5-7}$. Nesse contexto de pressão dentro do ambiente acadêmico, está cada vez mais e comum e preocupante o uso de bebidas alcoólicas pelos universitários a fim de aliviar sentimentos negativos e promover maior interação social ${ }^{8,9}$, o que pode reduzir a qualidade de sono e piorar o desempenho acadêmico do estudante ${ }^{10}$.

Além do uso de bebidas alcoólicas, os acadêmicos também fazem uso de substâncias psicoativas, principalmente a cafeína, para promover maior rendimento e alerta ao consumidor ${ }^{11}$. Visto que o consumo dessas bebidas energéticas poder estar ligado a ansiedade ${ }^{13}$, outros dois estudos ${ }^{13,14}$ demonstraram correlação estatisticamente significativa entre o uso das mesmas com a qualidade ruim de sono em estudantes universitários.

Poucos foram os estudos que tiveram como objetivo verificar a correlação entre o consumo de bebidas alcoólicas e/ou cafeína em acadêmicos universitários ${ }^{10,15-18}$, porém não há indícios na literatura brasileira sobre esse tema, especialmente em acadêmicos do curso de medicina. Portanto, este estudo teve como objetivo avaliar a qualidade de sono dos acadêmicos de medicina e analisar a possível correlação entre esta e o consumo de etanol e café.

\section{METODOLOGIA}

Trata-se de um estudo transversal, descritivo e quantitativo aprovado pelo comitê de ética em pesquisa da Faculdade de Medicina de Jundiaí (CEP - FMJ) por meio do parecer número 038657/2016. A população de estudo compreendeu os estudantes de medicina da Faculdade de Medicina de Jundiaí durante o período de aulas entre agosto e novembro de 2016 e foram inclusos aqueles maiores de 18 anos de idade regularmente matriculados na graduação e que não estivessem realizando algum tratamento para distúrbios do sono e a participação ocorreu somente depois de assinar o termo de consentimento livre e esclarecido. Foram excluídos os participantes que não responderam completamente os questionários utilizados.
Para o cálculo da amostra, foram utilizados os seguintes determinantes baseados no estudo de Zunhammer ${ }^{17}$ : desvio padrão do PSQI igual a 2,53, margem de erro absoluta igual a 0,51 (correspondendo ao coeficiente de variação do PSQI) e erro tipo I igual a 0,05 (5\%), resultando em uma amostra mínima de 95 participantes.

O questionário de Índice de Qualidade de Sono de Pittsburgh versão em português (PSQI-BR) foi utilizado para avaliar do ponto de vista psicológico a presença de sintomas de piora da qualidade de sono nos voluntários que participarão do estudo. O PSQI foi elaborado em 1989 e permite avaliar a qualidade do sono em relação ao último mês ${ }^{19}$.

O objetivo desse questionário é fornecer uma medida de qualidade de sono padronizada, fácil de ser respondida e interpretada. O questionário contém 19 questões que devem ser respondidas pelo próprio estudante e outras 5 questões que devem ser respondidas pelo seu companheiro de quarto, caso o tenha. Somente as 19 questões respondidas pelo estudante entraram na pontuação final e elas foram agrupadas em 7 componentes com pesos distribuídos numa escala de 0 a 3 . Os componentes são: qualidade subjetiva do sono, latência para o sono, duração do sono, eficiência habitual do sono, transtornos do sono, uso de medicamentos para dormir e a disfunção diurna. As pontuações desses componentes são somadas e o total varia de 0 a 21 pontos, no qual quanto maior a pontuação, pior a qualidade de sono ${ }^{19,20}$.

Para a avaliação geral de saúde e do consumo de café, foi anexado um questionário para avaliar o Índice de Massa Corpóreo (IMC) e o consumo de cafeína. O IMC $\left(\mathrm{Kg} / \mathrm{m}^{2}\right)$ é classificado pela Organização Mundial da Saúde $^{26}$ nas seguintes categorias: Baixo peso $(<18,5)$, Peso Normal (18,5-24,9), Sobrepeso (25-29,9), obesidade grau 1 (30 a 34,9), obesidade grau 2 (35 a 39,9) e obesidade grau $3(\geq 40)$. A mensuração da quantidade de café foi padronizada em xícaras de $50 \mathrm{~mL}$ e foi questionado quantas dessas os participantes haviam consumido diariamente nos últimos 30 dias $^{21}$. Em relação ao consumo de álcool, foi utilizado o questionário AUDIT, instrumento composto por 10 itens que abrangem três domínios teóricos: consumo de álcool, dependência do consumo de álcool e consequências adversas do uso de álcool nos últimos 12 meses. Sua pontuação varia de 0 a 40 pontos e um escore maior que pontos é sugestivo de dependência ${ }^{22}$. As doses de álcool no questionário foram baseadas no padrão da World Health Organization de $2004^{28}$, na qual 1 dose de álcool é equivalente a $350 \mathrm{~mL}$ de cerveja, $150 \mathrm{~mL}$ de vinho ou 40 $\mathrm{mL}$ de destilados.

Os resultados foram analisados utilizando-se o programa SPSS Statistics Base. Os resultados foram demonstrados como frequência absoluta e relativa em tabelas de contingência. Para a análise entre o índice de qualidade de sono e o padrão de consumo de álcool, café, IMC foi utilizado o teste qui-quadrado e as diferenças foram consideradas significativas quando $\mathrm{p}<0,05$. 


\section{RESULTADOS}

Os 3 questionários foram aplicados a 147 estudantes da Faculdade de Medicina de Jundiaí, de ambos os gêneros, em um único momento entre agosto e novembro de 2016. Ao todo, 5 estudantes tiveram os questionários excluídos

Tabela 1 - Características gerais dos participantes da pesquisa por não responderem todas as questões, restando, portanto, uma amostra de 143 estudantes, sendo 66 do $3^{\circ}$ ano, 44 do $2^{\circ}$ ano e 33 do $1^{\circ}$ ano, cujo predomínio foi do sexo feminino $(64,33 \%)$ e a média de idade dos participantes era de $22,41 \pm 2,84$ anos (Tabela 1 )

\begin{tabular}{c|ccc|c|c}
\hline Série & Homens (n) & Mulheres (n) & Idade média* \pm DP & Frequência absoluta & Porcentagem (\%) \\
\hline $\mathbf{1}^{\circ}$ & 9 & 24 & $20,61 \pm 2,13$ & 33 & 23,08 \\
$\mathbf{2}^{\circ}$ & 13 & 31 & $22,18 \pm 3,06$ & 44 & 30,77 \\
$\mathbf{3}^{\circ}$ & 29 & 37 & $23,49 \pm 2,53$ & 66 & 46,15 \\
Total & 51 & 92 & $22,41 \pm 2,84$ & 143 & 100 \\
\hline
\end{tabular}

Valores expressos em frequência seguida de sua porcentagem. *Valor médio seguido do desvio-padrão.

Os 143 voluntários, após responderem o PSQI-BR, foram categorizados em dois grupos: com somatória do questionário $\leq 5$ pontos e com somatória $>5$ pontos, sendo que esta última pontuação representa uma qualidade ruim de sono (24). $65,7 \%$ dos estudantes que responderam ao PSQI - BR obtiveram pontuação $>5$ (Tabela 2 ) e não houve diferença significativa quando se analisou qual ano teria pior índice de qualidade de sono $(\mathrm{p}=0,940)$.

Tabela 2 - Frequência dos resultados do PSQI em relação ao ano do estudante

\begin{tabular}{|c|c|c|c|c|c|c|}
\hline \multirow{2}{*}{$\begin{array}{l}\text { Pontuação PSQI-BR } \\
\text { (pontos) }\end{array}$} & \multicolumn{3}{|c|}{ Ano (frequência absoluta)* } & \multirow{2}{*}{$\begin{array}{c}\text { Frequência } \\
\text { absoluta }\end{array}$} & \multirow{2}{*}{$\begin{array}{c}\text { Porcentagem } \\
(\%)\end{array}$} & \multirow{2}{*}{$\mathbf{p}$} \\
\hline & $1^{\circ}$ & $2^{\circ}$ & $3^{\circ}$ & & & \\
\hline$\leq 5$ & 11 & 16 & 22 & 49 & 34,3 & \\
\hline$>5$ & 22 & 28 & 44 & 94 & 65,7 & 0.94 \\
\hline Total & 33 & 44 & 66 & 143 & 100 & \\
\hline
\end{tabular}

Os valores estão representados como valor absoluto. *Os resultados foram comparados utilizando o teste de qui- quadrado de Pearson e não houve diferença significativa entre os mesmos $(\mathrm{p}>0,05)$

Em relação ao questionário AUDIT, os voluntários foram categorizados em quatro grupos de padrão de consumo de álcool de acordo com a pontuação obtida (27): Consumo de Baixo Risco (0 a 7 pontos), Uso de Risco (8 a 15 pontos), Uso Nocivo (16 a 19 pontos) e
Provável Dependência ( $\geq 20$ pontos); $71,3 \%$ dos estudantes que responderam o questionário apresentaram consumo de baixo risco, enquanto que $21,7 \%$ dos voluntários demonstraram uso de risco de bebidas alcoólicas (Tabela 3).

Tabela 3 - Padrão do consumo de etanol, café e IMC dos estudantes da FMJ entre agosto e novembro de 2016

\begin{tabular}{|c|c|c|}
\hline Resultado AUDIT (pontos) & Frequência Absoluta & Porcentagem (\%) \\
\hline 0 a 7 & 102 & 71,3 \\
\hline 8 a 15 & 31 & 21,7 \\
\hline 16 a 19 & 6 & 4,2 \\
\hline$\geq 20$ & 4 & 2,8 \\
\hline \multicolumn{3}{|l|}{$\mathrm{IMC}\left(\mathrm{Kg} / \mathrm{m}^{2}\right)$} \\
\hline$<18,5^{*}$ & 6 & 4,2 \\
\hline $18,5-24,9^{* *}$ & 94 & 65,7 \\
\hline $25-29,9 * * *$ & 39 & 27,3 \\
\hline$>30 * * * *$ & 4 & 2,8 \\
\hline \multicolumn{3}{|l|}{ Consumo de café (doses) } \\
\hline Nenhuma & 37 & 25,9 \\
\hline 1 a 3 & 74 & 51,7 \\
\hline 4 a 6 & 16 & 11,2 \\
\hline 7 a 9 & 3 & 2,1 \\
\hline$>10$ & 13 & 9,1 \\
\hline Total & 143 & 100 \\
\hline
\end{tabular}


Pode-se perceber também que $65,7 \%$ dos estudantes estão com IMC dentro do índice normal (entre 18,5 e 24,9 $\mathrm{Kg} / \mathrm{m}^{2}$ ) e $51,7 \%$ dos alunos referiram beber de 1 a 3 doses de café por dia (Tabela 3), o equivalente a até 106,8 mg de cafeína por dia ${ }^{26}$.

A associação entre os resultados do PSQI - BR e do AUDIT, ambos categorizados, apresentaram coeficiente de correlação fraco $(\mathrm{R}=0,251)$, porém significativo $(\mathrm{p}=$ $0,02)$, resultando em ajuste linear (Gráfico 1). O teste de qui-quadrado de Pearson também resultou em $p=0,02$, o que indica associação entre maior consumo de álcool e qualidade de sono ruim (Tabela 4).

Gráfico 1 - Associação entre PSQI-BR e AUDIT

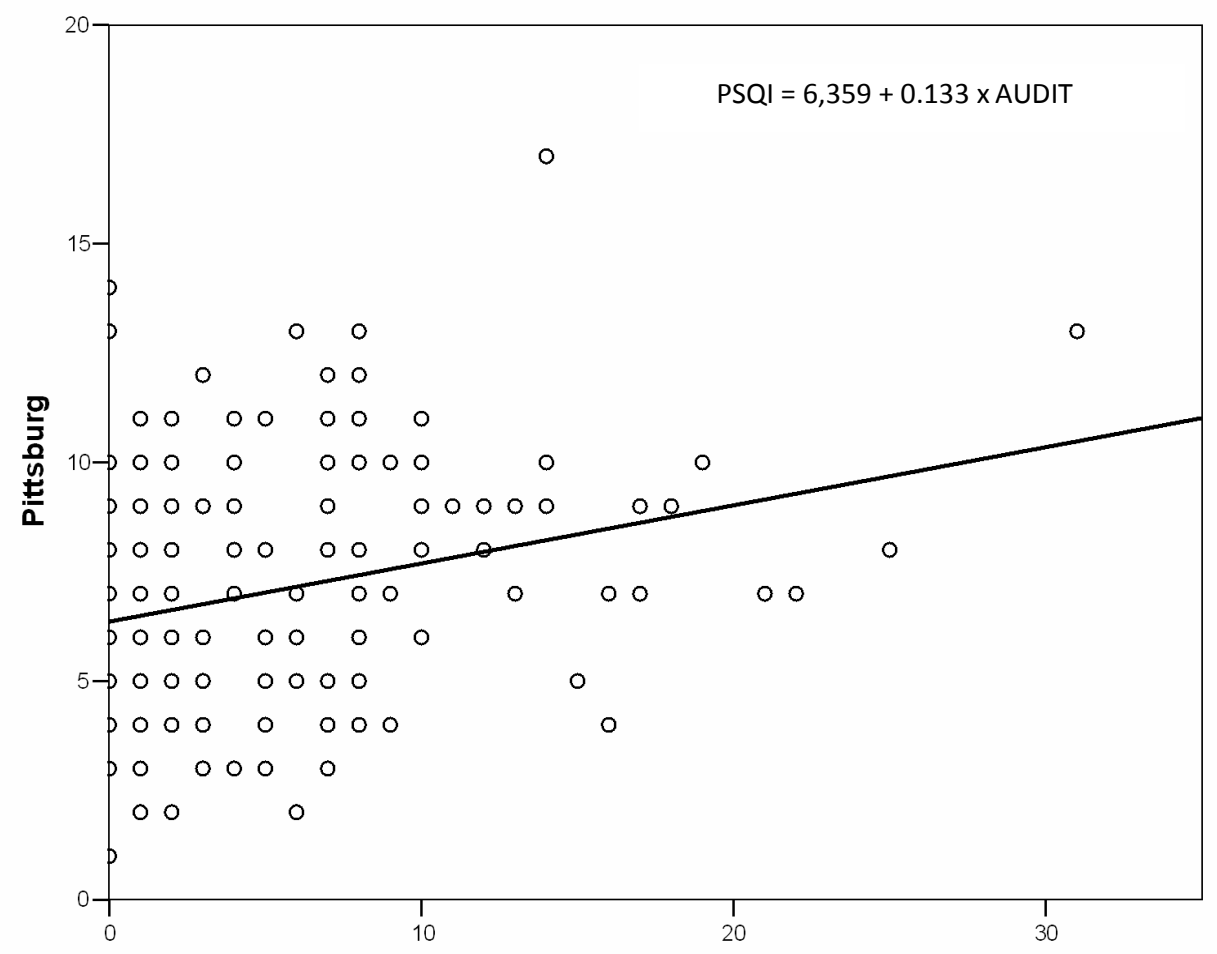

Gráfico de dispersão entre escore PSQI e AUDIT; $\mathrm{p}<0.05=0.251$

Em contrapartida, tanto a análise entre a qualidade de sono e o consumo de café quanto entre o primeiro e o IMC, analisados respectivamente pelo teste de Chiquadrado de Pearson e a correção de continuidade de Yates, resultou em $p=0.185$ para o primeiro e $p=0.391$ para o segundo (Tabela 4); Pode-se perceber, portanto que não há correlação significativa entre a qualidade de sono e o consumo de cafeína e IMC.

Tabela 4 - Correlação entre PSQI - BR e AUDIT, consumo de café e IMC

\begin{tabular}{|c|c|c|c|}
\hline & \multicolumn{2}{|c|}{ PSQI - BR (pontos) } & \multirow[b]{2}{*}{$\mathbf{p}$} \\
\hline & $\leq 5$ & $>5$ & \\
\hline \multicolumn{4}{|l|}{ AUDIT } \\
\hline 0 a 7 & 42 & 60 & \multirow{3}{*}{$0,02 *$} \\
\hline 8 a 15 & 6 & 25 & \\
\hline$\geq 16$ & 1 & 9 & \\
\hline \multicolumn{4}{|c|}{ Consumo de café (doses) } \\
\hline Nenhuma & 16 & 21 & \multirow{4}{*}{$0,185^{* *}$} \\
\hline 1 a 3 & 21 & 53 & \\
\hline 4 a 6 & 8 & 8 & \\
\hline$\geq 7$ & 4 & 12 & \\
\hline \multicolumn{4}{|l|}{$\operatorname{IMC}\left(\mathrm{Kg} / \mathrm{m}^{2}\right)$} \\
\hline$<25$ & 37 & 63 & \multirow{3}{*}{$0,391 * * *$} \\
\hline$\geq 25$ & 12 & 31 & \\
\hline Total & $49(34,3 \%)$ & $94(65,7 \%)$ & \\
\hline
\end{tabular}

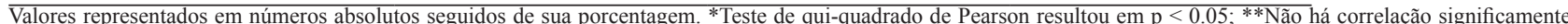
estatística através do teste de qui-quadrado de Pearson ( $\mathrm{p}>0.05$ ); ***Não há correlação significamente estatística através da Correção de Continuidade de Yates ( $\mathrm{p}>0.05$ ). 


\section{DISCUSSÃO}

Uma revisão sistemática publicada em 2005 sustentou o consenso científico de que o uso crônico de álcool tem interferência na fisiologia do sono, embora baixas doses possam promover inicialmente melhor sono ${ }^{27}$. Além disso, vários distúrbios psiquiátricos, como por exemplo, transtorno de ansiedade generalizada e depressão, também demonstraram relação com distúrbios de sono além do alcoolismo demonstrado por achados polissonográficos específicos ${ }^{28}$.

A vida acadêmica em faculdades e universidades é marcada por períodos de mudança, no qual a maioria dos jovens deve aprender a lidar com coerções sociais e inúmeras situações de estresse longe dos pais. A maioria das mudanças no estilo de vida dos estudantes inclui dieta não balanceada, uso de álcool, uso de tabaco, comportamentos sexuais inseguros e redução da carga horária de sono, o que pode levar a um detrimento do bem-estar dos estudantes ${ }^{24,25}$.

$\mathrm{O}$ atual estudo demonstrou até momento que $71,3 \%$ dos voluntários da Faculdade de Medicina de Jundiaí apresentam consumo de baixo risco de álcool, $92,3 \%$ não fumam, $51,7 \%$ consomem de 1 a 3 doses de café por dia e $65,7 \%$ destes mesmos estudantes demonstraram ter qualidade de sono ruim ( $>5$ pontos no PSQI-BR) entre agosto e novembro de 2016. Um estudo semelhante realizado na Universidade de Resenburg em 2014 demonstrou que $34,5 \%$ e $53,3 \%$ dos estudantes entrevistados obtiveram escore $>5$ pontos no PSQI antes e durante o período de exames, respectivamente; Todavia, este mesmo estudo demonstrou que o consumo de álcool não foi significativamente associado à qualidade de sono, ao contrário do nosso estudo que demonstrou uma fraca, porém significante correlação entre as variáveis; Apenas a não relação entre o consumo de cafeína e qualidade de sono foi de acordo com nosso trabalho ${ }^{17}$.

Em contrapartida, um estudo seccional em 6 universidades na Tailândia demonstrou que o consumo de pelo menos uma dose de bebida alcóolica eram mais propensos a relatar baixa qualidade de sono quando comparado aos estudantes que não bebiam. Os estudantes que consumiam mais de 19 doses de álcool por mês apresentaram o dobro de chances de ter baixa qualidade de sono em relação ao grupo que bebia menos de 1 dose de álcool por mês ${ }^{12}$. Esses resultados se apresentaram semelhantes ao atual estudo, visto que foi relatado que quanto maior o consumo de álcool, pior será a qualidade de sono do acadêmico de medicina.

Em relação ao índice de massa corpórea, $65,7 \%$ dos estudantes que responderam o questionário estão dentro do peso normal (entre 18,5 e $24,9 \mathrm{Kg} / \mathrm{m}^{2}$ ); nosso estudo não demonstrou associação significativa do IMC com a qualidade de sono. Outro estudo realizado em uma universidade no Sudoeste dos Estados Unidos entre outubro de 2010 e maio de 2011 demostrou que apenas o componente "Distúrbios de sono" do questionário PSQI foi um preditor significativo para sobrepeso/obesidade (IMC $\left.>25 \mathrm{Kg} / \mathrm{m}^{2}\right)^{15}$.

\section{CONCLUSÃo}

Nesse estudo fica demonstrada a baixa qualidade do sono dos estudantes da Faculdade de Medicina de Jundiaí e sua correlação com o uso de álcool, fortalecendo essa linha de raciocínio científica. Soma-se a esses distúrbios do sono, elevadas quantidades no consumo de cafeína e álcool, indicando a necessidade de recursos químicos pelos estudantes como ferramenta de suporte à estressante rotina acadêmica e existencial a que são submetidos.

Nesse sentido, esses fatores de risco precisam ser debatidos pela comunidade acadêmica e projetos que visem hábitos saudáveis e oportunidades de saúde aos estudantes de medicina devem ser promovidos e incentivados. $\mathrm{Na}$ realidade atual, dada a baixa qualidade de sono e o elevado consumo de cafeína e álcool, do ponto de vista pedagógico, diminui-se o rendimento cognitivo. No contexto discente, faz-se necessário um melhor equilíbrio entre a formação médica sólida e a qualidade de vida dos estudantes - meios indispensáveis a uma medicina efetiva e humanizada. Para aqueles que lidam com a vida humana, o autocuidado e as próprias condições de saúde mental e física são a única base onde a medicina pode ser aprendida e exercida de maneira verdadeira: buscando-se a saúde de todos, pacientes, alunos e médicos.

Conflitos de interesse: Não existem conflitos de interesse.

Participação dos autores: Gabriel Martinez: Elaboração do projeto, coleta de dados, redação ao artigo, análise de dados, elaboração das tabelas e gráficos, revisão, edição e submissão à revista. Heitor Castanharo Escãno: Coleta de dados, redação do artigo, revisão e edição. Maria Helena de Sousa: Análise dos dados, orientação e elaboração das tabelas e gráficos, revisão do texto. Clóvis Antônio Lopes Pinto: Orientação na elaboração do projeto, redação do artigo, análise de dados, revisão do texto e edição.

\section{REFERÊNCIAS}

1. Brown RE, Basheer R, McKenna JT, Strecker RE, McCarley RW. Control of sleep and wakefulness. Physiol Rev. 2012;92:1087-187. doi: 10.1152/physrev.00032.2011.

2. Araújo MFM, Freitas RWJF, Lima ACS, Pereira DCR, Zanetti ML, Damasceno MMC. Indicadores de saúde associados com a má qualidade do sono de universitários. Rev Esc Enferm USP. 2014;48(6):1085-92. doi: 10.1590/S0080623420140000700017.

3. Mellinger GD, Balter MB, Uhlenhuth EH. Insomnia and its treatment. Prevalence and correlates. Arch Gen Psychiatry. 1985; 42:225-32. http://dx.doi.org/10.1001/ archpsyc.1985.01790260019002. 
4. Leger D, Guilleminault C, Dreyfus JP, Delahaye C, Paillard M. Prevalence of insomnia in a survey of 12,778 adults in France. J Sleep Res. 2000;9:35-42. https://doi.org/10.1046/j.13652869.2000.00178.x.

5. Waqas A, Khan S, Sharif W, Khalid U, Ali A. Association of academic stress with sleeping difficulties in medical students of a Pakistani medical school: a cross sectional survey. Peer J. 2015;3:e840. doi: http://dx.doi.org/10.7717/peerj.840.

6. Ahrberg K, Dresler M, Niedermaier S, Steiger A, Genzel $\mathrm{L}$. The interaction between sleep quality and academic performance. J Psychiatr Res. 2012;46:1618-22. doi: http:// dx.doi.org/10.1016/j.jpsychires.2012.09.008.

7. Azad MC, Fraser K, Rumana N, Abdullah AF, Shahana N, Hanly PJ, Turin TC. Sleep disturbances among medical students: a global perspective. J Clin Sleep Med. 2015;11(1):69-74. doi: 10.5664/jcsm.4370.

8. Hingson RW, Zha W, Weitzman ER. Magnitude of and trends in alcohol-related mortality and morbidity among U.S. college students ages 18-24, 1998-2005. J Stud Alcohol Drugs Suppl. 2009;12-20. https://doi.org/10.15288/jsads.2009.s16.12.

9 Drinking Motives in the Prospective Prediction of Unique Alcohol-Related Consequences in College Students. J Stud Alcohol Drugs. 2014;93-102. https://doi.org/10.15288/ jsad.2014.75.93.

10. Van Reen E, Roane BM, Barker DH, McGeary JE, Borsari B, Carskadon MA. Current alcohol use is associated with sleep patterns in first-year college students. Sleep. 2016;39(6):13216. http://dx.doi.org/10.5665/sleep.5862.

11. Reissig CJ, Strain EC, Griffiths RR. Caffeinated energy drinks-a growing problem. Drug Alcohol Depend. 2009;99:1. doi: 10.1016/j.drugalcdep.2008.08.001.

12. Seifert SM, Schaechter JL, Hershorin ER, Lipshultz SE. Health effects of energy drinks on children, adolescents, and young adults. Pediatrics. 2011;127:511-28. doi: 10.1542/ peds.2009-3592.

13. Faris MAE, Jahrami H, Al-Hilali MM, et al. Energy drink consumption is associated with reduced sleep quality among college students: a cross-sectional study. Nutr Diet. 2017;74(3):268-74. doi: 10.1111/1747-0080.12289.

14. Lohsoonthorn V, Khidir H, Casillas G, et al. Sleep quality and sleep patterns in relation to consumption of energy drinks, caffeinated beverages and other stimulants among Thai College Students. Sleep Breath. 2013;17(3):1017-28. doi: 10.1007/s11325-012-0792-1.

15. Vargas PA, Flores M, Robles E. Sleep quality and body mass index in college students: the role of sleep disturbances. J Am Coll Health. 2014;62(8):534-41. doi:10.1080/07448481.201 4.933344

16. Rohsenow DJ, Howland J, Alvarez L, et al. Effects of caffeinated vs. non-caffeinated alcoholic beverage on nextday hangover incidence and severity, perceived sleep quality, and alertness. Addict Behav. 2014;39(1). doi:10.1016/j. addbeh.2013.09.008
17. Zunhammer M, Eichhammer P, Busch V. Sleep quality during exam stress: the role of alcohol, caffeine and nicotine. PLoS ONE. 2014;9(10):e109490. doi:10.1371/journal. pone. 0109490

18. Kenney SR, Paves AP, Grimaldi EM, LaBrie JW. Sleep quality and alcohol risk in college students: examining the moderating effects of drinking motives. J Am Coll Health. 2014;62(5):301-8. doi: 10.1080/07448481.2014.897953.

19. Buysse DJ, Reynolds CF, Monk TH, Berman SR, Kupfer DJ. The Pittsburg Sleep Quality Index: a New Instrument for Psychiatric Practice and Research. Psychiatry Res. 1989;28(2):193-213. doi: https://doi.org/10.1016/01651781(89)90047-4.

20. Bertolazi AN. Tradução, adaptação cultural e validação de dois instrumentos de avaliação de escala de sono: escala de sonolência de Epworth e Índice de Qualidade de Sono de Pittsburg [Dissertação]. Porto Alegre (RS): Universidade Federal do Rio Grande do Sul; 2008. http://hdl.handle. net/10183/14041.

21. Bunker ML, McWilliams M. Caffeine content of common beverages. J Am Diet Assoc. 1979;74:2832.

22. Santos WS, Fernandes DP, Grangeiro ASM, Lopes GS, Sousa EMP. Medindo consumo de álcool: análise fatorial confirmatória do Alcohol Use Disorder Identification Test (AUDIT). Psico-USF (Bragança Paulista). 2013;18(1):12130. http://dx.doi.org/10.1590/S1413-82712013000100013.

23. World Health Organization (WHO). Global Status Report on Alcohol 2004. Geneva: WHO; 2004. Disponível em: http:// www.who.int/substance abuse/publications/global status report_2004_overview.pdf.

24. Nacar M, Cetinkaya F, Baykan Z, Yilmazel G, Elmali F. Hazardous health behaviour among medical students: a study from Turkey. Asian Pac J Cancer Prev. 2015;16(17):7675-81. doi: http://dx.doi.org/10.7314/APJCP.2015.16.17.7675.

25. Brick CA, Seely DL, Palermo TM. Association between sleep hygiene and sleep quality in medical students. Behav Sleep Med. 2010;8(2):113-21. doi: 10.1080/15402001003622925.

26. World Health Organization. Obesity: preventing and managing the global epidemic. Report of a World Health Organization Consultation. Geneva: World Health Organization; 2000. p.256. (WHO Obesity Technical Report Series, n. 284). Disponível em: http://www.who.int/nutrition/publications/ obesity/WHO_TRS_894/en/.

27. Stein MD, Friedmann D. Disturbed sleep and its relationship to alcohol use. Subst Abus. 2005;26(1):1-13. https://doi. org/10.1300/J465v26n01_01.

28. Mendonça LL, Pradella-Hallinan M, Lucchesi M, Moraes WAS. O sono em transtornos psiquiátricos. Rev Bras Psiquiatr. 2005;27(Suppl 1):27-32. http://dx.doi.org/10.1590/ S1516-44462005000500006.

Submetido em: 04.03.18

Aceito em: 04.07.18 